\section{Transición del adolescente con enfermedad inflamatoria intestinal desde la atención pediátrica a la del adulto}

\author{
ELIZABETH NAVARRO ${ }^{1}$, JUAN C. OSSA ${ }^{2}$, \\ DANIELA SIMIAN $^{3}$, RODRIGO QUERA ${ }^{4}$
}

\section{Transition to adulthood of adolescents with inflammatory bowel disease}

Inflammatory bowel disease (IBD) is a prevalent chronic disorder, often diagnosed during childhood. Studies have suggested that the incidence of IBD in this group of patients is increasing. Children and adolescents with IBD frequently have more extensive and severe disease than adults. Transition is an important concept to ensure optimal health care management of adolescents and young adult patients with chronic physical and medical conditions. During this process there is a change in knowledge, attitudes and behavior towards the disease with a responsibility that gradually shifts from parents to the patient. The success of the transition process depends on the patient, pediatric and adult gastroenterologists. Thus, providers need to understand how to start, maintain and finish this process. When transition process is coordinated, staged and well planned, the adolescent and young adult will acquire the tools needed to successfully self-manage his or her own medical condition. Rather than a universal model of transition, each institution needs to adapt the most efficient model. The aim of this article is to review concepts pertinent to transition management for adolescents and young adults with IBD.

(Rev Med Chile 2015; 143: 767-773) care.

Key words: Adolescents; Inflammatory Bowel Diseases; Transition to adult

\author{
'Programa de Formación de \\ Especialista en Gastroenterología \\ Pediátrica, Escuela de Postgrado, \\ Facultad de Medicina, \\ Universidad de Chile. Servicio \\ de Pediatría, Hospital Exequiel \\ González Cortés. Departamento \\ de Pediatría y Cirugía Infantil \\ Campus Sur, Facultad de \\ Medicina, Universidad de Chile, \\ Santiago de Chile. \\ ${ }^{2}$ Sección de Gastroenterología, \\ Servicio de Pediatría, Hospital \\ Luis Calvo Mackenna, \\ Departamento de Pediatría y \\ Cirugía Oriente, Facultad de \\ Medicina, Universidad de Chile. \\ Departamento de Pediatría, \\ Unidad de Gastroenterología \\ Infantil, Clínica Las Condes, \\ Santiago de Chile. \\ ${ }^{3}$ Enfermera Sub Dirección \\ Académica, Clínica Las Condes, \\ Santiago de Chile. \\ ${ }^{4}$ Servicio de Gastroenterología. \\ Clínica Las Condes, Santiago de \\ Chile. \\ Trabajo apoyado por proyecto \\ Conicyt Redes No 130037; \\ Fondecyt $N^{\circ} 1110381$ (RQ) y \\ Fondecyt de Inicio No 11130374 \\ (JCO). \\ Recibido el 29 de septiembre de \\ 2014, aceptado el 17 de abril \\ de 2015. \\ Correspondencia a: \\ Dr. Rodrigo Quera \\ Teléfono: 26108749. \\ Fax: 26108719 \\ Estoril 450, Las Condes. \\ rquera@clc.cl
}

E ntendemos como transición al traslado planificado y organizado con fines concretos, del adolescente con una enfermedad crónica, desde un sistema de atención de salud orientado al niño hasta otro focalizado en la atención al adulto ${ }^{1}$. Constituye un proceso dinámico, programado, que requiere de tiempo; no implica sólo la confección de un resumen de los antecedentes del paciente y su derivación al especialista adulto, lo cual constituye el traslado ${ }^{2}$. Su objetivo es facilitar una atención continuada que incluya la normalización en el desarrollo social y emocional y la adquisición de habilidades necesarias para llevar una vida independiente ${ }^{3}$.
La importancia de los programas de transición se basa en dos aspectos fundamentales ${ }^{1}$ :

1. A medida que el adolescente se encamina hacia la fase de madurez, sus necesidades médicas, psicológicas y sociales cambian, por lo que la atención de salud debería también adaptarse y adecuarse.

2. Hay importantes diferencias entre la atención pediátrica y la del adulto (Tabla 1 ):

- En Pediatría: el cuidado intenta ser multidisciplinario, se focaliza en la familia, quedando la toma de decisiones y el tratamiento a cargo de los padres, teniendo el niño/adolescente un papel pasivo, con poca autonomía. Existe 
Tabla 1. Diferencias en el manejo de los pacientes con Enfermedad Inflamatoria Intestinal según visión del Gastroenterólogo Infantil y Adulto (Adaptado de Martin de Carpi ${ }^{1}$ )

\begin{tabular}{|ll|}
\hline Manejo gastroenterólogo infantil & Manejo gastroenterólogo adulto \\
\hline Centrado en el crecimiento, pubertad y nutrición & $\begin{array}{l}\text { Centrado en el riesgo durante el embarazo, vigilancia neo- } \\
\text { plasias, nuevos fármacos }\end{array}$ \\
\hline Endoscopia: apoyo con anestesia (propofol) & $\begin{array}{l}\text { Endoscopia: mayoría de las ocasiones con sedación (mida- } \\
\text { zolam) }\end{array}$ \\
\hline $\begin{array}{l}\text { Orientado en la familia } \\
\text { El niño es un paciente especial. Existe un acercamiento al ado al paciente, padres no son considerados en la } \\
\text { niño }\end{array}$ & $\begin{array}{l}\text { Orientadente es uno más, relación neutral } \\
\text { toma de decisiones }\end{array}$ \\
$\begin{array}{l}\text { Actitud del médico: acoger, dedica más tiempo a la evolución } \\
\text { y atención de los pacientes }\end{array}$ & $\begin{array}{l}\text { Actitud del médico: más distante, dedica menos tiempo a la } \\
\text { evaluación de los pacientes }\end{array}$ \\
\hline
\end{tabular}

mayor habilidad en la detección y manejo del retraso del crecimiento, además preocupación por el beneficio versus riesgo de estudios de laboratorio, por el inicio de la experimentación sexual, alcohol, tabaco y drogas y por la continuidad de la escolaridad.

- En adultos: el cuidado se centra en el paciente, que debe tomar sus decisiones y hacerse cargo de su tratamiento, las consultas suelen ser más breves y rápidas. Hay mayor vigilancia de condiciones secundarias como el cáncer, osteoporosis y preocupación por la fertilidad y la suspensión del hábito tabáquico.

Los períodos de transición son críticos para la evolución de las enfermedades crónicas, ya que al dejar la atención pediátrica, muchos pacientes abandonan los controles médicos, llevando a un mal control de la enfermedad, falta de adherencia con suspensión de los medicamentos y aumento de la tasa de ingresos hospitalarios por descompensación ${ }^{4,5}$.

A pesar de conocer la importancia de un proceso ordenado de transición en las enfermedades crónicas, existen pocos datos de los efectos de un programa establecido en pacientes con EII, midiendo resultados clínicamente relevantes, como utilización de servicios de urgencia, adherencia a medicamentos, tasas de complicaciones e indicadores de calidad de vida ${ }^{6}$. El objetivo de este artículo es revisar el proceso de transición en la EII, más allá del tratamiento farmacológico, con el fin de mejorar la calidad de atención de salud y de vida de estos pacientes.

\section{Evolución de la EII en pediatría}

El 15 a $20 \%$ de los casos de EII se diagnostica antes de los 20 años $^{6-10}$, constituyéndose en una de las enfermedades digestivas más importantes a la que se enfrenta el gastroenterólogo infantil. Los pacientes diagnosticados a menor edad tienden a tener un compromiso más extenso al debut y un curso más severo de la enfermedad, comparado con aquellos que la inician en edad adulta ${ }^{11}$.

La colitis ulcerosa (CU) clásicamente compromete desde el recto hacia proximal con una extensión variable; diversas cohortes muestran que la colitis extensa es la forma de presentación más frecuente $e^{4,5,11}$.

En la enfermedad de Crohn (EC), el compromiso puede afectar cualquier segmento de la boca al ano. En pacientes pediátricos el compromiso de la región ileocolónica es la localización más frecuente, teniendo más de la mitad de los pacientes compromiso del tracto gastrointestinal superior ${ }^{4,5,11}$.

El impacto nutricional de la EII en la población pediátrica es constante, el bajo peso se presenta en forma característica en $85 \%$ de los niños con EC $\mathrm{y}$ al menos en $65 \%$ de los pacientes con $\mathrm{CU}^{5,6}$. La interferencia con el desarrollo puberal en pacientes que debutan con la enfermedad tanto en estadio prepuberal como puberal, se debe principalmente al fracaso en inducir la remisión y como consecuencia del compromiso nutricional y niveles elevados de citoquinas proinflamatorias (como IL-6 y TNF- $\alpha$ ), las cuales influenciarían proteínas claves en el crecimiento, como el factor de crecimiento insulínico tipo 1 (IGF-1) ${ }^{4}$. También estos niños 
presentan déficit de micronutrientes tales como hierro, vitaminas D y B12, calcio y zinc. Estudios han descrito niveles bajos de vitamina $\mathrm{D}$ hasta en $60 \%$ de la población estudiada ${ }^{12}$. Por esto, es necesario prestar atención a los aspectos nutricionales y del crecimiento, con estricto seguimiento de las curvas de peso, talla y desarrollo puberal.

El tratamiento médico busca inducir la remisión y luego mantenerla. En forma resumida, en la EC dentro de los tratamientos para inducir remisión están la nutrición enteral, los corticoesteroides y la terapia biológica (infliximab/adalimumab/certolizumab); para mantención drogas inmunosupresoras como las tiopurinas (azatioprina/6-mercaptopurina) o metotrexato y también terapia biológica ${ }^{12-15}$. En CU, mesalazina/5-ASA derivados, corticoesteroides y terapia biológica pueden ser indicados en la fase de inducción; para mantener la remisión se pueden utilizar tiopurinas y biológicos ${ }^{13,14,16,17}$. Las opciones terapéuticas se consideran dependiendo del contexto del paciente y su familia, el patrón clínico de la enfermedad, las complicaciones y la disponibilidad de tratamiento. También es necesario evaluar los potenciales eventos adversos y los beneficios de cada terapia.

Pese a los avances en la terapia farmacológica, algunos pacientes requerirán cirugía. En la EC, esta estrategia tiene como objetivo el manejo de las complicaciones (estenosis, fístulas, enfermedad perianal o refractariedad a terapia farmacológica), describiéndose tasas de cirugías de $20 \%$ a los 3 años del debut y $34 \%$ a los 5 años ${ }^{18}$. En CU, si bien la colectomía remueve por completo el foco de la enfermedad, mejorando la calidad de vida y evitando los potenciales riesgos del tratamiento inmunosupresor, cabe mencionar que otras complicaciones pueden desarrollarse, como el compromiso del reservorio, incontinencia o afectación de la fertilidad ${ }^{19}$.

El éxito de la terapia depende de mantener el tratamiento por largo tiempo. Tal como en otras enfermedades crónicas, la falta de adherencia al tratamiento varía entre 38 y $66 \%$ en niños con $\mathrm{EII}^{5}$, pudiendo ser menores en adolescentes. Este es un problema de difícil manejo, pues existen diversos factores involucrados (familiares, educacionales y psicológicos), por lo que las estrategias para mejorar la adherencia también son múltiples, como educar al paciente, estimular el desarrollo de autonomía e intentar manejo de factores familiares y psicosociales.
Otro punto crítico en niños y adolescentes es la evaluación psicosocial, debido a que padecen de una enfermedad crónica compleja. Aspectos como escolaridad, actividades sociales y trastornos del ánimo deben ser evaluados constantemente, pues se ven afectados, sobretodo en cursos más severos e inestables de la enfermedad. Los adolescentes habitualmente se sienten vulnerables, diferentes, con poco control sobre sus vidas y dudas sobre su futuro. Se han descrito tasas de depresión tan altas como en $25 \%$ de los pacientes ${ }^{5}$, siendo frecuentemente subestimada por familiares y por el equipo médico.

En el proceso de transición, el paciente además debe comprender y adaptarse a cambios en los objetivos que busca el clínico, desde lograr un adecuado crecimiento y desarrollo en el cuidado pediátrico, a la preocupación en aspectos como sexualidad, fertilidad y cáncer que enfatiza el adulto ${ }^{2}$.

\section{EII y neoplasias}

Los niños y adolescentes con EII están en riesgo de desarrollar neoplasias a largo plazo dado la temprana edad del diagnóstico de la enfermedad, la extensión y severidad de ésta y el tiempo de exposición a agentes inmunosupresores ${ }^{20}$. Los pacientes con EII tienen un mayor riesgo de cáncer colorrectal asociado a menor edad al diagnóstico, enfermedad de larga data, mayor severidad y extensión, presencia de colangitis esclerosante primaria y antecedentes familiares de cáncer colorrectal $^{21}$. Aunque en Pediatría, el cáncer colorrectal es una complicación infrecuente, es importante la vigilancia, ya que después de 10 años de diagnosticada la EII el riesgo aumenta gradualmente, variando de una probabilidad de $4,4 \%$ a los 10 años, $8,6 \%$ a los 20 años, hasta alcanzar $43 \%$ a los 35 años del diagnóstico ${ }^{22}$.

Los pacientes con EII presentan un mayor riesgo de desarrollar otras neoplasias, como cáncer de piel $^{23}$, siendo similar a aquellos sometidos a trasplantes de órganos sólidos que usan terapia inmunosupresora. El riesgo de desarrollar cáncer de piel no melanoma y melanoma, aumenta con el uso de tiopurinas y terapia biológica, respectivamente $^{23,24}$. Por lo tanto, las recomendaciones del uso de protección solar y de evaluaciones dermatológicas rutinarias son importantes en estos pacientes. 
También se ha señalado que pacientes con EII tienen mayor riesgo de desarrollar linfoma, principalmente asociado al uso de tiopurinas ${ }^{23,24}$. Este riesgo podría aumentar en pacientes con serología positiva para virus Epstein Barr ${ }^{23}$. Existe un tipo de linfoma no Hodgkin, no relacionado con la presencia de este virus, más agresivo y resistente a quimioterapia, el linfoma T hepatoesplénico, cuya incidencia se ha visto aumentada. Se han reportado aproximadamente 200 casos a nivel mundial, de los cuales 36 corresponden a adolescentes con EII en tratamiento con tiopurinas monoterapia o asociado a biológicos ${ }^{23,24}$.

En resumen, los niños que inician tratamiento inmunosupresor tienen un riesgo acumulado para el desarrollo de patología maligna dado el tiempo de evolución de la enfermedad, siendo de vital importancia la identificación de los factores de riesgo y una adecuada vigilancia de estas condiciones en la niñez y adolescencia.

\section{EII y fertilidad}

En cuanto a la fertilidad, se ha planteado que podría estar reducida por aspectos psicológicos, secundario a procedimientos quirúrgicos y a la actividad de la enfermedad ${ }^{25}$. Dentro del primer punto, destaca la preocupación por el embarazo y posibles riesgos de la terapia farmacológica. Por otro lado, la colectomía total y la creación del reservorio provocan disminución de la fertilidad, probablemente debido a adherencias en la pelvis u obstrucción secundaria de las trompas de Falopio $^{25}$. En pacientes sin antecedentes de cirugía, la fertilidad también puede verse afectada por la actividad de la enfermedad ${ }^{26}$. En remisión, el embarazo tendría un efecto beneficioso en cuanto a la evolución de la EII, en cambio, en pacientes con EII activa, existe un mayor riesgo de una evolución desfavorable ${ }^{26,27}$. Respecto a los fármacos, sólo metotrexato y talidomida deben suspenderse en el embarazo ${ }^{25-28}$. La terapia biológica es segura, traspasa la placenta con niveles detectables en el recién nacido hasta los 6 meses, lo que no ha demostrado tener efectos deletéreos, recomendándose solamente evitar las vacunas vivas en este período de tiempo ${ }^{26,29}$.

La EII afecta en su gran mayoría a población en etapa fértil, por lo que el equipo tratante debe lograr que el paciente adolescente entienda los efectos que la enfermedad y el tratamiento podrían tener sobre la fertilidad.

\section{Proceso de transición en pacientes pediátricos con EII}

En la EII, la mayor parte de los estudios sobre este proceso se basan en opiniones de expertos, encuestas y estudios descriptivos ${ }^{1}$. En el año 2002 se establecieron recomendaciones a seguir para crear un adecuado programa de transición en EII, destacando que el proceso se debe iniciar al comenzar la adolescencia, entre los 10 y 12 años, dándole gradualmente independencia y autonomía al paciente, conversando con él o ella y su familia en relación a los beneficios de la transición, logrando una relación cercana con el gastroenterólogo de adultos, facilitando toda la información médica y resúmenes necesarios ${ }^{3}$.

El paciente es el protagonista de este proceso, esperándose que durante este período adquiera las habilidades para su autocuidado y autonomía en la toma de decisiones. Se han desarrollado múltiples herramientas para evaluar el proceso de transición, como encuestas (TRAQ, SMART) que se pueden usar en diferentes patologías crónicas y otras específicas para EII, como "Mi pasaporte de Salud", "IBD yourself" y "Lista chequeable de Fishman", que buscan evaluar las competencias que idealmente deben adquirir los pacientes previo a su traslado, aunque ninguna de ellas ha sido validada hasta la fecha ${ }^{30-33}$. Estos instrumentos pueden ayudarnos a detectar áreas específicas en las que el paciente requiere atención, facilitando la programación de intervenciones, logrando una mejor transición al cuidado del adulto.

Estudios han demostrado que la adquisición de habilidades de autocuidado, relacionadas al conocimiento y manejo de la enfermedad, medicamentos y controles médicos, mejoran a mayor edad del paciente asociado a una mayor madurez; independiente del tiempo de evolución y severidad de la enfermedad ${ }^{34}$. Los jóvenes que han sido diagnosticados a menor edad suelen presentar una enfermedad más severa, no obstante se involucran menos en las decisiones médicas de su enfermedad, quedando esa responsabilidad en sus padres, por lo que requieren mayor apoyo y educación para una transición exitosa ${ }^{35}$. Por otro lado, los adolescentes tienen menor adherencia a controles y tratamiento que los adultos ${ }^{30}$, de ahí la 
importancia de un correcto proceso de transición.

En estudios que han intentado analizar la forma en que se transfieren los pacientes y las limitaciones existentes en este proceso, en base a encuestas realizadas tanto a gastroenterólogos infantiles y adultos como a adolescentes con la enfermedad, se ha encontrado diferentes tipos de barreras en el proceso. En relación al paciente, se encuentran la falta de autonomía, de conocimiento de la enfermedad y del tratamiento ${ }^{8}$, además manifiestan dificultades en el aspecto logístico, como solicitud de horas médicas, encontrar un especialista de adultos para continuar su cuidado y dificultades con su previsión de salud ${ }^{35}$. Desde el punto de vista de los gastroenterólogos, tanto pediátricos como de adultos, concuerdan que la edad de transición puede ser flexible, teniendo los especialistas infantiles la función de introducir tempranamente el concepto en los pacientes para completar el proceso a los 18 años. Los médicos tratantes pediátricos y adultos estiman que 35\% de los pacientes tiene insuficiente conocimiento acerca de su condición y tratamiento al momento de la transferencia ${ }^{8}$, datos obtenidos de gastroenterólogos de adultos reportan que 55\% de ellos muestra déficit en conocimiento de su historia médica y $69 \%$ en sus medicamentos ${ }^{35}$. Por otro lado, los gastroenterólogos pediátricos estiman que existe falta de coordinación en el proceso en $34 \%$ de los $\operatorname{casos}^{2}$ y muestran mayor interés en participar en unidades de transición que los adultos.

Finalmente, en relación a la organización de los sistemas de salud, los problemas observados son falta de tiempo de los involucrados, de espacio, de financiamiento y de apoyo de otros servicios de soporte como enfermería, considerando que una pequeña cantidad de pacientes necesitaría someterse al proceso de transición en forma regular ${ }^{8}$.

Debido a la falta de estudios clínicos aleatorios, no hay un modelo único de transición y no se ha estudiado, hasta la fecha, cuál es el más apropiado, por lo que cada institución se adapta, dependiendo de los recursos, localización geográfica, cantidad de pacientes y disponibilidad de especialistas adultos e infantiles ${ }^{2}$. Dentro de los modelos están la programación de visitas conjuntas, única o múltiples, entre el equipo infantil y adulto, y la creación de una consulta específica de transición, que tiene como objetivo la preparación del adolescente para el traslado, cuya coordinación habitualmente está dada por una enfermera especializada en $\mathrm{EII}^{36}$.
Entrevistas a jóvenes con EII han mostrado que más de $20 \%$ de los mayores de 18 años de edad ha necesitado algún tipo de ayuda en diversas tareas para manejar adecuadamente su enfermedad ${ }^{34}$, lo cual nos confirma la necesidad de intervención y educación por el equipo de salud, pudiendo involucrar otros profesionales, como asistentes sociales y psicólogos.

La transición se produce en diferentes momentos dependiendo del país, en Europa y Canadá debe producirse la transferencia a los 18 años, en cambio, en Estados Unidos de Norteamérica, los pacientes pueden mantenerse en el seguro médico de sus padres hasta los 26 años, lo cual disminuye su sensación de autonomía ${ }^{2}$. En Chile, la atención pediátrica termina a los 15 años, sin embargo, frente a patologías crónicas el traslado suele retrasarse. Lo ideal es evaluar al adolescente progresivamente sin sus padres para construir una adecuada relación médico-paciente, que promueva su independencia y confianza. El equipo de trabajo debe estar formado por un gastroenterólogo de adultos que tenga experiencia en el manejo de pacientes jóvenes con EII que hayan debutado en la infancia. El gastroenterólogo infantil debe otorgar el máximo de antecedentes para permitir una adecuada atención en el futuro y preparar al paciente y su familia para la transición, discutiendo con ellos los beneficios de este proceso.

Es importante considerar que la transferencia se debe llevar a cabo idealmente con la enfermedad estable, si hay una recaída en este período debería manejarse en conjunto con el equipo adulto que continuará el manejo, sobre todo si hay necesidad de cambio de fármacos o cirugía ${ }^{2}$.

Nosotros proponemos que para obtener un proceso de transición favorable en nuestro país, éste debiera iniciarse entre los 12 y 14 años, con visitas conjuntas por el equipo infantil y de adultos. El equipo pediátrico debe ir otorgando gradualmente más autonomía al paciente, con tiempo de visitas sin sus padres. En esta instancia podría aplicarse algún tipo de cuestionario abreviado, de respuestas simples, como la lista usada por Whitfield et $\mathrm{al}^{34}$, para así poder establecer las áreas a trabajar con el paciente en evaluaciones conjuntas entre el gastroenterólogo infantil y el de adultos, las cuales se podrían iniciar a partir de los 14 años, lo que permitiría alcanzar finalmente una transferencia exitosa, a los 18 años (Figura 1). 


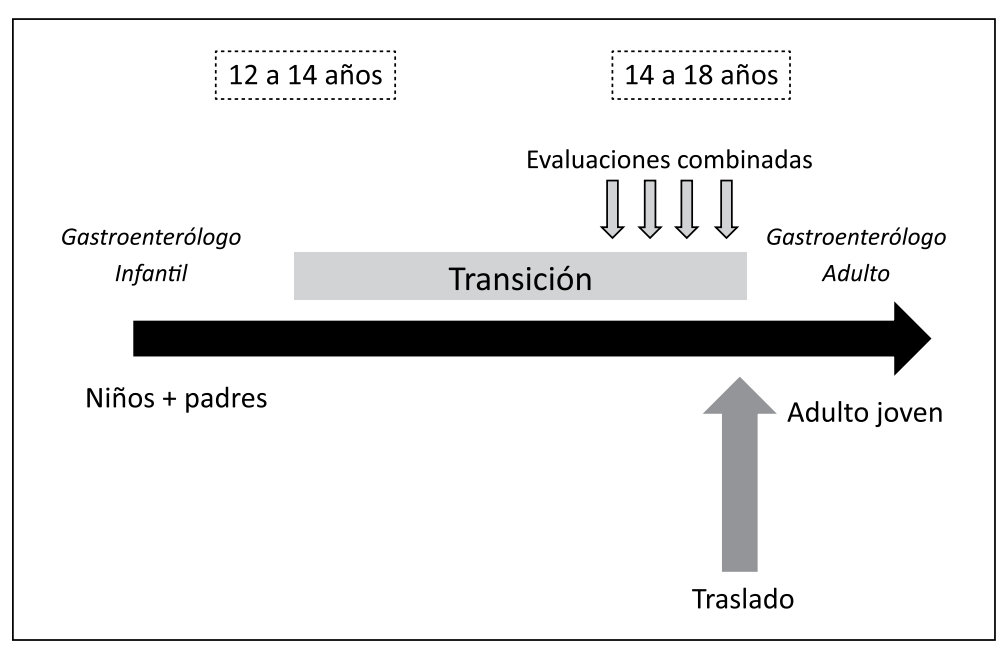

Figura 1. Transición en el manejo de los pacientes con enfermedad inflamatoria intestinal.

\section{Conclusiones}

El período de la adolescencia y de transición a los cuidados del adulto constituyen una fase crítica en el manejo de las enfermedades crónicas que debutan en la infancia, por lo que los programas de transición deberían establecerse como un continuo de atención, adaptándose a los cambios psicológicos, físicos y sociales propios de esta etapa de la vida.

Dado el aumento que ha mostrado la EII pediátrica, es importante el desarrollo de programas organizados de transición, que cuenten con gastroenterólogos infantiles y de adultos, en unidades especializadas de EII, dado que el manejo, las condiciones clínicas y las complicaciones de la enfermedad son diferentes en cada etapa de la vida. Esto constituye un gran desafío en nuestro país, donde no existe un programa establecido de transición, por lo que las derivaciones siguen siendo la confección de un resumen e interconsultas que llevan los mismos pacientes y no un proceso que culmina con un paciente capaz de manejar su enfermedad con responsabilidad y autonomía.

\section{Referencias}

1. Martín de Carpi J. Transición a los cuidados del adulto. Enfermedad Inflamatoria Intestinal al día 2012; 11: 187 95.

2. de Silva PS, Fishman LN. Transition of the patient with IBD from pediatric to adult care-an assessment of $\mathrm{cu}-$ rrent evidence. Inflamm Bowel Dis 2014; 20: 1458-64.

3. Baldassano R, Ferry G, Griffiths A, Mack D, Markowitz J, Winter H. Transition of the patient with inflammatory bowel disease from pediatric to adult care: recommendations of the North American Society for Pediatric Gastroenterology, Hepatology and Nutrition. J Pediatr Gastroenterol Nutr 2002; 34: 245-8.

4. Day A, Ledder O, Leach S, Lemberg D. Crohn`s and colitis in children and adolescents. World J Gastroenterol 2012; 18: 5862-9.

5. Bishop J, Lemberg DA, Day A. Managing inflammatory bowel disease in adolescent patients. Adolesc Health Med Ther 2014; 5: 1-13.

6. Zeisler B, Hyams JS. Transition of management in adolescents with IBD. Nat Rev Gastroenterol Hepatol 2014; 11: 109-15.

7. Leung Y, Heyman MB, Mahadevan U. Transitioning the adolescent inflammatory bowel disease patient: guidelines for the adult and pediatric gastroenterologist. Inflamm Bowel Dis 2011; 17: 2169-73.

8. Sebastián S, Jenkins H, McCartney S, Ahmad T, Arnott I, Croft N, et al. The requirements and barriers to successful transition of adolescents with inflammatory bowel disease: differing perceptions from a survey of adult and pediatric gastroenterologists. J Crohns Colitis 2012; 6: 830-44.

9. Benchimol EI, Manuel DG, Guttmann A, Nguyen GC, Mojaverian N, Quach P, et al. Changing age demographics of inflammatory bowel disease in Ontario, Canada: a population-based cohort study of epidemiology trends. Inflamm Bowel Dis 2014; 20: 1761-9.

10. Kim SC, Ferry GD. Inflammatory bowel diseases in 
pediatric and adolescent patients: Clinical, therapeutic, and psychological considerations. Gastroenterology 2004; 126, 1550-60.

11. Gower-Rousseau C, Vasseur F, Fumery M, Savoye G, Salleron J, Dauchet L, et al. Epidemiology of inflammatory bowel diseases: new insights from a French population-based registry (EPIMAD). Dig Liver Dis 2013; 45: 89-94.

12. Levin AD, Wadhera V, Leach ST, Woodhead HJ, Lemberg DA, Mendoza-Cruz AC, et al. Vitamin D deficiency in children with inflammatory bowel disease. Dig Dis Sci 2011; 56: 830-6.

13. Mahadevan U, Cucchiara S, Hyams JS, Steinwurtz F, Nuti F, Travis SPL. The London position statement of the World Congress of Gastroenterology on biological therapy for IBD with the European Crohn's and Colitis Organization: pregnancy and pediatrics. Am J Gastroenterol 2011; 106: 214-23.

14. Aloi M, Nuti F, Stronati L, Cucchiara S. Advances in the medical management of paediatric IBD. Nat Rev Gastroenterol 2014; 11: 98-108.

15. Singh S, Pardi D. Update on anti-Tumor necrosis Factor agents in Crohn Disease. Gastroenterol Clin N Am 2014; 43: 457-78.

16. Dignass A, Lindsay JO, Sturm A, Windsor A, Colombel JF, Allez M, et al. J Crohns Colitis 2012; 6: 991-1030.

17. Samaan MA, Bagi P, Casteele NV, D'Haens GR, Levesque BG. An update on anti-TNF agents in Ulcerative Colitis. Gastroenterol Clin N Am 2014; 43: 479-94.

18. Vernier-Massouille G, Balde M, Salleron J, Turck D, Dupas JL, Mouterde O, et al. Natural history of pediatric Crohn's disease: a population based cohort study. Gastroenterology 2008; 135: 1106-13.

19. Turner D. Relapsing and refractory ulcerative colitis in children. Dig Dis 2014; 32: 419-26.

20. Connell W, Wilson J. Cancer Surveillance in Inflammatory Bowel Disease. En "Inflammatory bowel disease: translating basic science into clinical practice”. Edited by Stephan R. Targan, Fergus Shanahan, Loren C. Karp. Wiley-Blackwell. Singapore. 2010.

21. Kim ER, Chang DK. Colorectal cancer in inflammatory bowel disease: The risk, pathogenesis, prevention and diagnosis. World J Gastroenterol 2014; 20: 9872-81.

22. Eaden JA, Abrams KR, Mayberry JF. The risk of colorectal cancer in ulcerative colitis: a meta-analysis. Gut 2001; 48: 526-35.

23. Dulai PS, Siegel CA. The risk of malignancy associated with the use of biological agents in patients with inflammatory bowel disease. Gastroenterol Clin North Am 2014; 43: 525-41.

24. Rufo PA. Inflammatory bowel disease and neoplasia in children. Dig Dis 2014; 32: 455-62.

25. Palomba S, Sereni G, Falbo A, Beltrami M, Lombardini $\mathrm{S}$, Boni MC, et al. Inflammatory bowel diseases and human reproduction: a comprehensive evidence-based review. World J Gastroenterol 2014; 20: 7123-36.

26. Horst $S$, Kane $S$. The use of biologic agents in pregnancy and breastfeeding. Gastroenterol Clin North Am 2014; 43: 495-508.

27. Cury DB, Moss AC. Treatment of Crohn`s disease in pregnant women: drug and multidisciplinary approaches. World J Gastroenterol 2014; 20: 8790-5.

28. Nielsen $\mathrm{OH}$, Maxwell C, Hendel J. IBD medications during pregnancyo and lactation. Nat Rev Gastroenterol Hepatol 2014; 11: 116-27.

29. Mahadevan U. Fertility and Pregnancy in Inflammatory Bowel Disease. En "Inflammatory bowel disease: translating basic science into clinical practice". Edited by Stephan R. Targan, Fergus Shanahan, Loren C. Karp. Willey-Blackwell. Singapore. 2010.

30. Paine CW, Stollon NB, Lucas MS, Brumley LD, Poole ES, Peyton T, et al. Barriers and facilitators to successful transition from pediatric to adult Inflammatory Bowel Disease care from the perspectives of providers. Inflamm Bowel Dis 2014; 20: 2083-91.

31. Hait E, Arnold JH, Fishman LN. Educate, communicate, anticipate-practical recommendations for transitioning adolescents with IBD to adult health care. Inflamm Bowel Dis 2006; 12: 70-3.

32. Benchimol EI, Walters TD, Kaufman M, Frost K, Fiedler $\mathrm{K}$, Chinea Z, et al. Assessment of knowledge in adolescents with inflammatory bowel disease using a novel transition tool. Inflamm Bowel Dis 2011; 17: 1131-7.

33. Zijlstra M, De Bie Ch, Breij L, van Pieterson $M$, van Staa A, de Ridder L, et al. Self-efficacy in adolescents with inflammatory bowel disease: A pilot study of the "IBD-yourself", a disease-specific questionnaire. J Crohns Colitis 2013; 7: e375-e85.

34. Whitfield EP, Fredericks EM, Eder SJ, Shpeen BH, Adler J. Transition readiness in pediatric patients with inflammatory bowel disease: patient survey of self-management skills. J Pediatr Gastroenterol Nutr 2015; 60: 36-41.

35. Plevinsky JM, Gumidyala AP, Fishman LN. Transition experience of young adults with inflammatory bowel disease (IBD): a mixed methods study. Child Care Health Dev 2014; 6: 1-7.

36. Golik M, Kurek M, Poteralska A, Bieniek E, Marynka A, Pabich G, et al. Working Group guidelines on the nursing roles in caring for patients with Crohn`s disease and ulcerative colitis in Poland. Prz Gastroenterol 2014; 9 (4): 179-93. 